\title{
Mental Simulation of Action in the Service of Action Perception
}

\author{
Vassilis Raos, Mina N. Evangeliou, and Helen E. Savaki \\ Department of Basic Sciences, Faculty of Medicine, School of Health Sciences, University of Crete, Iraklion, 71003 Crete, Greece, and Institute of Applied \\ and Computational Mathematics, Foundation for Research and Technology-Hellas, 71110 Iraklion, Crete, Greece
}

We used the quantitative ${ }^{14} \mathrm{C}$-deoxyglucose method to map the activity pattern throughout the frontal cortex of rhesus monkeys, which either grasped a three-dimensional object or observed the same grasping movements executed by a human. We found that virtually the same frontal cortical networks were recruited for the generation and the perception of action, including the primary motor cortex (MI/F1), premotor cortical areas (F2, F5, and F6), the primary (SI) and supplementary (SSA) somatosensory cortex, medial cortical areas $(8 \mathrm{~m}$ and $9 \mathrm{~m})$, and the anterior cingulate. The overlapping networks for action execution and action observation support the notion that mental simulation of action could underlie the perception of others' actions. We suggest that the premotor and the somatotopic MI/F1 activations induced by action observation reflect motor grasp of the observed action, whereas the somatotopic SI and the SSA activations reflect recruitment of learned sensory-motor associations enabling perceptual understanding of the anticipated somatosensory feedback. We also found that the premotor activations were stronger for action observation, in contrast to the primary somatosensory-motor ones, which were stronger for action execution, and that activations induced by observation were bilateral, whereas those induced by execution were contralateral to the moving forelimb. We suggest that these differences in intensity and lateralization of activations between the executive and the perceptual networks help attribute the action to the correct agent, i.e., to the "self" during action execution and to the "other" during action observation. Accordingly, the "sense of agency" could be articulated within the core components of the circuitry supporting action execution/observation.

Key words: action observation; action recognition; mental simulation; motor cortex; premotor cortex; somatosensory cortex

\section{Introduction}

The premotor cortical area F5 contains "mirror neurons," which discharge both when a monkey performs an object-related hand action and when the monkey observes another individual performing the same action, and could thus be responsible for the capacity of individuals to recognize actions made by others (Gallese et al., 1996; Rizzolatti et al., 1996). However, the resonant system that helps match action perception to action generation encompasses much more of the cortex than the mirror neuron concept would lead one to believe. We recently found that the forelimb regions of the primary motor (MI/F1) and somatosensory (SI) cortices within the central sulcus (Cs) are activated when subjects observe object-related hand actions, and they are activated somatotopically as they are for execution of the same actions (Raos et al., 2004).

The aim of the present study was to explore whether frontal areas other than MI/F1 and F5 are also involved in action obser-

Received July 1, 2007; revised Aug. 16, 2007; accepted 0ct. 1, 2007.

This work was supported by the European Union (FP6 Grant IST-027574) and the General Secretariat of Research and Technology, Hellas (Grant 01ED111). We thank A. K. Moschovakis for constructive comments, G. G. Gregoriou for advice and help regarding histology, and M. Kefaloyianni for technical assistance.

Correspondence should be addressed to Helen E. Savaki, Department of Basic Sciences, Faculty of Medicine, School of Health Sciences, University of Crete, P.0. Box 2208, Iraklion, GR-71003 Crete, Greece. E-mail: savaki@med.uoc.gr.

D01:10.1523/JNEUROSCI.2988-07.2007

Copyright $\odot 2007$ Society for Neuroscience ～0270-6474/07/2712675-09\$15.00/0 vation. We used the $\left[{ }^{14} \mathrm{C}\right]$-deoxyglucose $\left({ }^{14} \mathrm{C}\right.$-DG) quantitative autoradiographic method (Sokoloff et al., 1977) to obtain highresolution functional images of the monkey frontal and cingulate cortical areas activated for grasping execution and grasping observation. The ${ }^{14} \mathrm{C}$-DG method is the only imaging approach to offer the following advantages: (1) assessment of brain activity directly and not indirectly via blood flow changes, (2) quantitative measurement of glucose consumption instead of semiquantitative relative differences, (3) resolution of $20 \mu \mathrm{m}$, and (4) cytoarchitectonic identification of cortical areas in sections adjacent to the autoradiographic ones.

We examined the lateral premotor cortex, including the dorsal areas F2 and F7 and the ventral areas F4 and F5 (Matelli et al., 1991; Geyer et al., 2000), as well as the medial premotor cortex, including the supplementary motor areas F3 or SMA-proper and F6 or pre-SMA, as well as the rostral (CMAr), dorsal (CMAd), and ventral (CMAv) cingulate motor areas (Matelli et al., 1991; He et al., 1995). We also examined additional medial cortical areas such as the 8-medial $(8 \mathrm{~m})$, 9-medial $(9 \mathrm{~m}), 24,23$, and the supplementary somatosensory area (SSA) (Murray and Coulter, 1981; Morecraft et al., 2004). Histological examination of the brain sections enabled us to assign most of the activated regions of the reconstructed metabolic maps to cytoarchitectonically defined areas of the frontal lobe.

We found that, far from being restricted to frontal areas F5, $\mathrm{MI} / \mathrm{F} 1$, and SI, the so-called "action observation/execution 
matching system" also involves extensive regions of both the lateral- and medial-frontal cortex. Because, as shown here, nearly the same widespread frontal and cingulate cortical circuits are recruited for both action perception and action generation, and because the mental simulation theory assigns the role of perceiving others' actions to the neural substrate that is also responsible for action execution, our data suggest that "mental simulation" of actions rather than "mirroring" (Goldman and Sebanz, 2005) better accounts for the recognition of actions performed by others.

\section{Materials and Methods}

Subjects and behavioral tasks. Six head-fixed adult female monkeys ( $\mathrm{Ma}$ caca mulatta) weighing between 3 and $5 \mathrm{~kg}$ were used. Experiments were approved by the institutional animal use committee in accordance with the European Council Directive 86/609/EEC. A detailed description of surgical procedures, behavioral apparatus, EMG, and eye position recording was reported previously (Raos et al., 2004). In brief, a sliding window at the front side of the behavioral apparatus allowed the subject (monkey or experimenter) to grasp a horizontally oriented ring with the index finger inserted into it (with the hand pronated) while eye movements were recorded (see Fig. 1) with an infrared oculometer (Dr. Bouis, Karlsruhe, Germany). All monkeys were trained for several months before the ${ }^{14} \mathrm{C}$-DG experiment to perform their tasks continuously for at least $1 \mathrm{~h}$ per day. On the day of the ${ }^{14} \mathrm{C}-\mathrm{DG}$ experiment, monkeys performed their tasks for the entire ${ }^{14} \mathrm{C}$-DG experimental period (45 min) without any breaks, and successful completion of each trial was rewarded with water.

The arm-motion control $(\mathrm{Cm})$ monkey had to maintain its gaze straight ahead for 2.7-3 s, during the opening of the window of the behavioral apparatus, the presentation of the illuminated object behind the window, and the closure of the window, and while the experimenter was reaching with extended hand toward the closed window. Thus, this control monkey was used to exclude not only the potential effects of the biological motion of the reaching forelimb, but also the possible effects of unspecific arousal and attention, gaze fixation, visual stimulation by the three-dimensional (3D) object, and eye movements used to scan the object. The intertrial intervals ranged between 2 and $2.5 \mathrm{~s}$. During the training and the ${ }^{14} \mathrm{C}-\mathrm{DG}$ experiment both forelimbs of this monkey were restricted.

Two grasping-execution (E) monkeys were trained to reach and grasp with their left forelimbs while the right ones were restricted. These monkeys had to fixate the object for $0.7-1 \mathrm{~s}$, until a dimming of the light would signal reaching, grasping, and pulling the ring with the left forelimb (within $1 \mathrm{~s}$ ) while maintaining fixation (intertrial intervals: 2-2.5 s).

Three grasping-observation $(\mathrm{O})$ monkeys were first trained to perform the task of the E monkeys and then trained to observe the same grasping movements executed by the experimenter. Both forelimbs of the $\mathrm{O}$ monkeys were restricted during the observation training and during the ${ }^{14} \mathrm{C}$-DG experiment. Although grasping training took place months before the ${ }^{14} \mathrm{C}$-DG experiment, to cancel any possible side-toside effects caused by this earlier grasping training, the first monkey was trained to grasp with its left hand, the second one with its right hand, and the third one with both hands consecutively. The experimenter was always standing on the right side of the monkey and was using the right arm/hand for reaching/grasping. Both reaching and grasping components of the movement were visible to the monkey. Object and movement parameters were similar to the ones described for the E task. To control for possible rate-related effects, the mean rate of movements was set to be similar for the arm-motion control, the execution, and the observation tasks.

Reconstruction of two-dimensional maps of activity. We used the ${ }^{14} \mathrm{C}-\mathrm{DG}$ quantitative autoradiographic method (Sokoloff et al., 1977) to obtain high-resolution functional images of the monkey frontal and cingulate cortical areas activated for grasping execution and grasping observation. The ${ }^{14} \mathrm{C}-\mathrm{DG}$ experiment and the brain tissue processing for autoradiography were performed as described previously (Savaki et al., 1993; Raos et al., 2004). In brief, the tracer was injected intravenously 5 min after initiation of task performance, and arterial blood samples were drawn for the next $45 \mathrm{~min}$ as per the original description of the method (Sokoloff et al., 1977). Plasma glucose levels, blood pressure, hematocrit, and blood gases ranged within normal values in all monkeys and remained constant throughout the ${ }^{14} \mathrm{C}$-DG experiment. Glucose utilization values (in micromoles per $100 \mathrm{~g}$ per minute) were calculated from the original operational equation of the method (Sokoloff et al., 1977).

To cover the full extent of the lateral-frontal cortex of interest, $\sim 1000$ serial horizontal sections of $20 \mu \mathrm{m}$ thickness were used from each hemisphere of each monkey, and 800 sections were used for the reconstruction of the medial-frontal cortex. For each horizontal section, a data array was obtained by sampling the local cerebral glucose utilization (LCGU) values along a rostrocaudal line parallel to the surface of the cortex and covering all cortical layers (anteroposterior sampling spatial resolution, $50 \mu \mathrm{m} / \mathrm{pixel}$ ). Every five adjacent sections of $20 \mu \mathrm{m}$, data arrays were averaged and plotted to produce one line in the $2 \mathrm{D}$ maps of activity (spatial resolution of plots, $100 \mu \mathrm{m}$ ). The anterior crown of the Cs was used for the alignment of adjacent data arrays in the lateral cortex, and the anterior tip of the brain was used for alignment of the medial cortex. One section every $500 \mu \mathrm{m}$ was stained with thionine for identification of the cytoarchitectonic borders of sensory-motor and premotor cortical areas (Matelli et al., 1991; Geyer et al., 2000; Gregoriou et al., 2005). Tick marks in each horizontal section labeling (1) surface landmarks of the brain (such as crown, fundus, and tip) and (2) cytoarchitectonically identified borders of cortical areas of interest were used to match the 2D maps obtained from different hemispheres and animals (see geometrical normalization of maps, below). Normalization of LCGU values was based on the averaged unaffected gray matter value pooled across all monkeys (Savaki et al., 1993).

Geometrical normalization of the two-dimensional maps of activity. The geometrical normalization of the individual 2D maps of LCGU values (glucograms) in the lateral and medial cortices was based on combined cytoarchitectonic and surface landmarks and was generated as follows.

In the dorsal portion of all lateral-frontal cortical maps, the sectionby-section distances between (1) the anterior cytoarchitectonic border of F7 and the anterior border of F2, (2) the latter and the anterior border of $\mathrm{MI} / \mathrm{F} 1,(3)$ the latter and the anterior crown of the Cs (point of alignment), (4) the latter and the fundus of Cs, and (5) the fundus and the posterior crown of the Cs were measured. The average of these measures was computed to produce a reference map of landmarks (see Fig. $2 b$, dorsal segment). The areal surface in $\mathrm{mm}^{2} \pm \mathrm{SE}$ was $166.7 \pm 6.4$ for F1/MI bank, $97.6 \pm 11.1$ for F1/MI convexity, $164.7 \pm 8.1$ for SI bank, $75.2 \pm 6.7$ for F2, and $32.4 \pm 2.9$ for F7. The reference map of landmarks in the ventral portion of the lateral-frontal cortex was generated similarly. The distances used here were those between (1) the fundus of the arcuate sulcus (As) and its crown, (2) the latter and the posterior border of F5, (3) the latter and the anterior crown of the Cs, (4) the latter and the fundus of Cs, and (5) the fundus and the posterior crown of Cs (see Fig. $2 b$, ventral segment). The areal surface in $\mathrm{mm}^{2} \pm \mathrm{SE}$ was $21.3 \pm 2.8$ for F5 convexity, $81.1 \pm 4.7$ for F5 bank, and $37.7 \pm 2.3$ for F4. Each individual lateral-frontal cortical map with its own dorsal and ventral segments' landmarks was linearly transformed in Matlab (MathWorks, Natick, MA) to match the reference map. This allowed us to overlay functional and cytoarchitectonic maps from all hemispheres of all monkeys to obtain averaged maps per case and to subtract control from experimental maps.

In the dorsal portion of the medial-frontal convexity, the distances used to produce the reference map of landmarks were those between (1) the anterior tip of the brain (point of alignment) and the anterior border of F3, (2) the latter and the anterior border of MI/F1, (3) the latter and the anterior border of SI, (4) the latter and the dorsal crown of the cingulate sulcus (Cgs) (see Fig. 3b, above the dorsal crown of the Cgs). The areal surface was $45.3 \pm 4.3$ for SI, $46.1 \pm 1.9$ for F1, $46.4 \pm 1.9$ for F3, and $46.7 \pm 3.2$ for F6. The distance used to generate the reference map of landmarks in the dorsal bank of the Cgs was that between the dorsal crown of the Cgs and its fundus, separately for its anterior, middle, and posterior segments (see Fig. $3 b$ ). The corresponding areal surfaces were $69.5 \pm 3.9,79.2 \pm 5$, and $36.5 \pm 3.9$. The distance used to produce the reference map of landmarks in the ventral bank of the Cgs was that 
between the fundus and the ventral crown of the Cgs, separately for its anterior, middle, and posterior segments (see Fig. $3 b$ ). The corresponding areal surfaces were $48.5 \pm 2,74.2 \pm 3.8$, and $29.4 \pm 3$. The distance used to produce the reference map of landmarks in the ventral convexity was that between the ventral crown of the Cgs and (1) the extension of the posterior tip of Cgs for the dorsalmost sections or (2) the corpus callosum for the ventralmost sections. The corresponding areal surfaces were $191.3 \pm 6$ and $86.2 \pm 4.5$, respectively. Although its total surface changed considerably when an area was geometrically normalized, the intensity and the spatial distribution of LCGU effects were preserved within each single area because these effects were proportionally shrunk or expanded within its borders.

Statistical analysis. The average LCGU values were calculated in sets of five adjacent sections (20 $\mu \mathrm{m}$ thick) throughout each cortical area of interest in each hemisphere. Experimental to control LCGU values were compared for statistical significances by the Student's unpaired $t$ test. Given that ipsilateral to contralateral LCGU values in normal control monkeys range up to 7\% (Kennedy et al., 1978), only differences $>7 \%$ were considered for statistical treatment. The percentage LCGU differences between the experimental ( $\mathrm{E}$ and $\mathrm{O}$ ) and the $\mathrm{Cm}$ monkeys were generated using the formulas $(\mathrm{E}-\mathrm{Cm}) / \mathrm{Cm} \times 100$ and $(\mathrm{O}-\mathrm{Cm}) / \mathrm{Cm} \times$ 100.

\section{Results}

During the critical first $10 \mathrm{~min}$ of the ${ }^{14} \mathrm{C}$-DG experiment, the $\mathrm{Cm}$ monkey observed nine movements of the experimenter's arm per minute. The dwell time of the line of sight of the $\mathrm{Cm}$ monkey in different eye positions during the critical first $10 \mathrm{~min}$ of the experiment is illustrated in Figure 1a. Because we found no significant side-to-side difference of glucose consumption in the $\mathrm{Cm}$ monkey (see LCGU values for left and right hemispheres separately in Table 1), the quantitative glucograms (quantitative maps of LCGU) of the lateral (Fig. 2c) and the medial-frontal and cingulate (Fig. $3 c$ ) cortices of one side were averaged with the corresponding ones of the other side.

The E monkeys performed an average of 10 grasping movements per minute during the critical first 10 min of the ${ }^{14} \mathrm{C}-\mathrm{DG}$ experiment. The dwell time of the line of sight in different eye positions during the critical first $10 \mathrm{~min}$ of the ${ }^{14} \mathrm{C}-\mathrm{DG}$ experiment averaged over the two E monkeys is shown in Figure $1 b$. We generated LCGU maps of the lateral (Fig. 2d) and the medial (Fig. $3 d$ ) cortices by averaging the two corresponding, geometrically normalized glucograms in the right hemispheres (contralateral to the moving forelimb) of the two E monkeys. The latter glucograms as well as the equivalent ones in the left hemispheres (ipsilateral to the moving forelimb) were used for measurement of the LCGU values in cortical areas of interest, their statistical comparisons, and the estimation of the percentage differences from the Cm control values (Table 1).

To illustrate the percentage LCGU differences between the $\mathrm{E}$ monkeys and the $\mathrm{Cm}$, we generated images using the formula $(\mathrm{E}-\mathrm{Cm}) / \mathrm{Cm} \times 100$ for each one of the lateral and the medial cortical maps. When the averaged maps (lateral or medial) of the right hemispheres of the E monkeys are compared with the averaged maps of the $\mathrm{Cm}$ monkey, increased metabolic activity (net activation) is apparent in several frontal and cingulate cortical regions. Areas activated for execution of grasping movements include the proximal forelimb representation of F1-convexity, F2-forelimb region, F5-bank, and F5-convexity, in addition to the forelimb representations in the SI and the MI/F1 of the Cs (Fig. 4a, Table 1). They also include several medial areas such as the F3/SMA-proper, F6/pre-SMA, CMAd, CMAv, CMAr, $8 \mathrm{~m}$, $9 \mathrm{~m}, 24,23$, and SSA (Fig. 5a, Table 1). Most of the activated regions were found in the hemisphere contralateral to the grasping hand (Table 1).
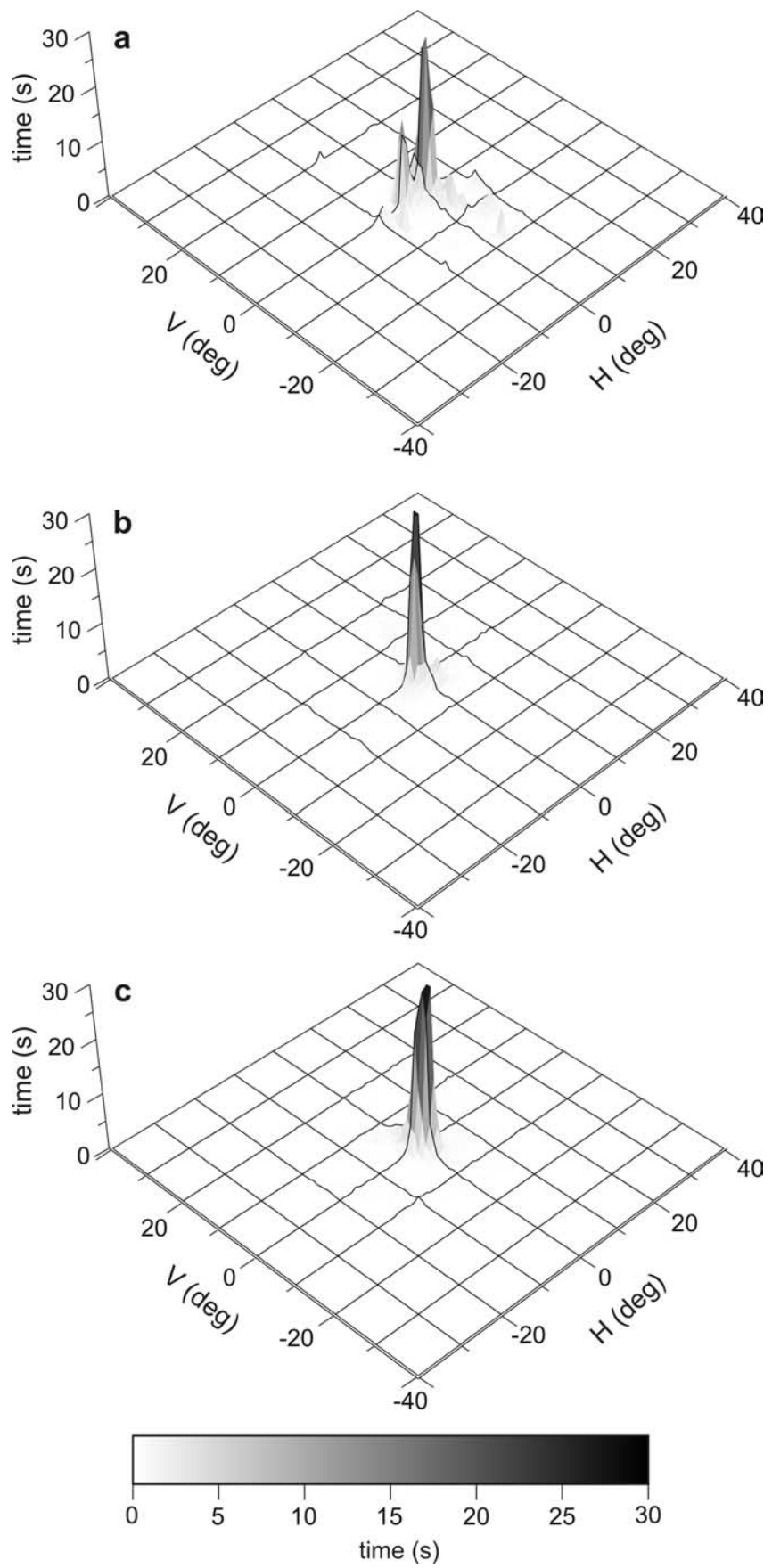

Figure 1. Three-dimensional histograms of the dwell time of the line of sight as a function of eye position during the critical first $10 \mathrm{~min}$ of the ${ }^{14} \mathrm{C}-\mathrm{DG}$ experiment. $\boldsymbol{a}$, Motion-control monkey. $\boldsymbol{b}$, Averaged oculomotor behavior from the two grasping-execution monkeys. $\boldsymbol{c}$, Averaged behavior from the three grasping-observation monkeys. Horizontal axis $(H ; X)$ and vertical axis $(V ; y)$ in degrees, $z$-axis in seconds. Grayscale bar indicates time in seconds.

The $\mathrm{O}$ monkeys observed an average of 12 grasping movements per minute during the critical first $10 \mathrm{~min}$ of the ${ }^{14} \mathrm{C}-\mathrm{DG}$ experiment. The dwell time of the line of sight in different eye positions during the critical first $10 \mathrm{~min}$ of the experiment averaged over the three $\mathrm{O}$ monkeys is shown in Figure $1 c$. When the averaged maps of the lateral (Fig. $2 e$ ) and the medial and cingulate (Fig. $3 e$ ) cortex of the right hemispheres of the three $\mathrm{O}$ monkeys are compared with the averaged Cm maps (Figs. $2 c, 3 c$ ), regions of increased metabolic activity included lateral-frontal areas, such as the F7, F2-forelimb, F5-bank, and F5-convexity, in 
Table 1. Metabolic effects in frontal cortical areas of the monkey brain

\begin{tabular}{|c|c|c|c|c|c|c|c|c|c|c|c|c|}
\hline Cortical area & $n$ & $\begin{array}{l}\mathrm{Cml} \\
\mathrm{LCGU} \\
\pm \mathrm{SD}\end{array}$ & $\begin{array}{l}\mathrm{Cmr} \\
\mathrm{LCGU} \\
\pm \mathrm{SD}\end{array}$ & $\begin{array}{l}\mathrm{Cm} \\
\mathrm{LCGU} \\
\pm \mathrm{SD}\end{array}$ & $\begin{array}{l}\text { EILCGU } \\
\pm S D\end{array}$ & $\begin{array}{l}\text { ErLCGU } \\
\pm S D\end{array}$ & $\begin{array}{l}\text { OI LCGU } \\
\pm \text { SD }\end{array}$ & $\begin{array}{l}\text { OrLCGU } \\
\pm S D\end{array}$ & $\mathrm{El} / \mathrm{Cm}(\%)$ & $\mathrm{Er} / \mathrm{Cm}(\%)$ & $0 \mathrm{l} / \mathrm{Cm}(\%)$ & $\mathrm{Or} / \mathrm{Cm}(\%)$ \\
\hline \multicolumn{13}{|c|}{ Primary somatosensory and motor areas } \\
\hline SI-forelimb (central sulcus) & 98 & $62 \pm 2$ & $61 \pm 2$ & $61 \pm 2$ & $62 \pm 3$ & $76 \pm 5$ & $64 \pm 3$ & $68 \pm 3$ & 2 & 25 & 5 & 11 \\
\hline $\begin{array}{l}\text { SI-forelimb (max, central } \\
\text { sulcus) }\end{array}$ & 24 & $63 \pm 3$ & $58 \pm 1$ & $60 \pm 2$ & $61 \pm 2$ & $77 \pm 3$ & $69 \pm 1$ & $72 \pm 2$ & 2 & 28 & 15 & 20 \\
\hline SI-trunk (central sulcus) & 80 & $66 \pm 4$ & $67 \pm 3$ & $66 \pm 3$ & $69 \pm 4$ & $75 \pm 4$ & $70 \pm 5$ & $70 \pm 6$ & 5 & 14 & 6 & 6 \\
\hline SI-hindlimb (medial cortex) & 65 & $59 \pm 3$ & $57 \pm 2$ & $58 \pm 2$ & $58 \pm 3$ & $59 \pm 2$ & $57 \pm 2$ & $58 \pm 2$ & 0 & 2 & -2 & 0 \\
\hline F1-forelimb (central sulcus) & 103 & $58 \pm 3$ & $55 \pm 3$ & $56 \pm 3$ & $55 \pm 4$ & $66 \pm 3$ & $58 \pm 4$ & $61 \pm 5$ & -2 & 18 & 4 & 9 \\
\hline $\begin{array}{l}\text { F1-forelimb (max, central } \\
\text { sulcus) }\end{array}$ & 28 & $59 \pm 2$ & $54 \pm 1$ & $57 \pm 1$ & $58 \pm 1$ & $71 \pm 2$ & $61 \pm 1$ & $63 \pm 1$ & 2 & 25 & 7 & 11 \\
\hline F1-forelimb convexity & 65 & $51 \pm 1$ & $51 \pm 2$ & $51 \pm 1$ & $50 \pm 2$ & $58 \pm 2$ & $50 \pm 1$ & $54 \pm 2$ & -2 & 14 & -2 & 6 \\
\hline F1-trunk (central sulcus) & 80 & $63 \pm 6$ & $63 \pm 4$ & $63 \pm 4$ & $61 \pm 2$ & $65 \pm 3$ & $67 \pm 7$ & $65 \pm 5$ & -3 & 3 & 6 & 3 \\
\hline F1-trunk convexity & 42 & $51 \pm 2$ & $51 \pm 3$ & $51 \pm 2$ & $53 \pm 2$ & $54 \pm 1$ & $52 \pm 1$ & $52 \pm 1$ & 4 & 6 & 2 & 2 \\
\hline F1-hindlimb (medial cortex) & 65 & $52 \pm 4$ & $52 \pm 4$ & $52 \pm 3$ & $54 \pm 3$ & $54 \pm 4$ & $52 \pm 2$ & $52 \pm 2$ & 4 & 4 & 0 & 0 \\
\hline \multicolumn{13}{|l|}{ Lateral premotor areas (dorsal) } \\
\hline F7 & 60 & $44 \pm 2$ & $45 \pm 2$ & $45 \pm 1$ & $44 \pm 5$ & $47 \pm 4$ & $49 \pm 2$ & $50 \pm 1$ & -2 & 4 & 9 & 11 \\
\hline F2-forelimb & 48 & $45 \pm 3$ & $46 \pm 3$ & $45 \pm 3$ & $46 \pm 2$ & $51 \pm 2$ & $49 \pm 2$ & $52 \pm 2$ & 2 & 13 & 9 & 16 \\
\hline F2-forelimb convexity & 47 & $45 \pm 2$ & $46 \pm 3$ & $46 \pm 3$ & $46 \pm 2$ & $51 \pm 1$ & $48 \pm 1$ & $52 \pm 3$ & 0 & 11 & 4 & 13 \\
\hline F2-forelimb bank & 32 & $45 \pm 4$ & $45 \pm 3$ & $45 \pm 3$ & $44 \pm 3$ & $51 \pm 3$ & $50 \pm 2$ & $54 \pm 1$ & -2 & 13 & 11 & 20 \\
\hline F2-hindlimb convexity & 42 & $53 \pm 6$ & $51 \pm 4$ & $52 \pm 4$ & $53 \pm 6$ & $55 \pm 4$ & $52 \pm 2$ & $50 \pm 2$ & 2 & 6 & 0 & -4 \\
\hline \multicolumn{13}{|l|}{ Lateral premotor areas (ventral) } \\
\hline F5 bank & 98 & $55 \pm 4$ & $53 \pm 3$ & $54 \pm 2$ & $56 \pm 3$ & $59 \pm 3$ & $62 \pm 3$ & $62 \pm 2$ & 4 & 9 & 15 & 15 \\
\hline F5 bank dorsal & 40 & $54 \pm 5$ & $52 \pm 3$ & $53 \pm 3$ & $53 \pm 1$ & $56 \pm 2$ & $59 \pm 1$ & $61 \pm 2$ & 0 & 6 & 11 & 15 \\
\hline F5 bank ventral & 58 & $55 \pm 3$ & $52 \pm 3$ & $54 \pm 2$ & $58 \pm 2$ & $61 \pm 1$ & $64 \pm 2$ & $62 \pm 2$ & 7 & 13 & 19 & 15 \\
\hline F5 convexity & 65 & $56 \pm 5$ & $55 \pm 5$ & $55 \pm 4$ & $61 \pm 5$ & $62 \pm 2$ & $59 \pm 2$ & $59 \pm 2$ & 11 & 13 & 7 & 7 \\
\hline F5 convexity (max) & 33 & $55 \pm 2$ & $50 \pm 4$ & $53 \pm 2$ & $64 \pm 4$ & $64 \pm 1$ & $59 \pm 1$ & $59 \pm 2$ & 21 & 21 & 11 & 11 \\
\hline F4 & 76 & $68 \pm 4$ & $64 \pm 3$ & $66 \pm 3$ & $65 \pm 5$ & $65 \pm 5$ & $65 \pm 3$ & $67 \pm 4$ & -2 & -2 & -2 & 2 \\
\hline \multicolumn{13}{|c|}{ Medial cortical areas (dorsal convexity) } \\
\hline $9 m$ convexity anterior & 49 & $41 \pm 1$ & $40 \pm 3$ & $41 \pm 2$ & $48 \pm 1$ & $50 \pm 1$ & $43 \pm 1$ & $45 \pm 2$ & 17 & 22 & 5 & 10 \\
\hline $9 \mathrm{~m}$ convexity posterior & 22 & $46 \pm 2$ & $47 \pm 2$ & $47 \pm 1$ & $49 \pm 1$ & $50 \pm 1$ & $47 \pm 1$ & $46 \pm 1$ & 4 & 6 & 0 & -2 \\
\hline $8 m(\max )$ & 24 & $47 \pm 2$ & $49 \pm 2$ & $48 \pm 1$ & $53 \pm 2$ & $57 \pm 4$ & $55 \pm 1$ & $52 \pm 1$ & 10 & 19 & 15 & 8 \\
\hline F6/pre-SMA (max) & 24 & $56 \pm 5$ & $54 \pm 1$ & $55 \pm 2$ & $58 \pm 1$ & $62 \pm 3$ & $60 \pm 1$ & $58 \pm 3$ & 5 & 13 & 9 & 5 \\
\hline F3/SMA-proper (max) & 31 & $51 \pm 1$ & $49 \pm 3$ & $50 \pm 2$ & $51 \pm 2$ & $54 \pm 1$ & $52 \pm 1$ & $53 \pm 1$ & 2 & 8 & 4 & 6 \\
\hline \multicolumn{13}{|l|}{ Cingulate sulcus (dorsal bank) } \\
\hline $24 \mathrm{~d}$ anterior & 43 & $41 \pm 3$ & $41 \pm 3$ & $41 \pm 2$ & $47 \pm 2$ & $50 \pm 2$ & $49 \pm 2$ & $48 \pm 2$ & 15 & 22 & 20 & 17 \\
\hline 24d middle & 41 & $57 \pm 3$ & $55 \pm 3$ & $56 \pm 2$ & $56 \pm 3$ & $50 \pm 4$ & $55 \pm 2$ & $51 \pm 1$ & 0 & -11 & -2 & -9 \\
\hline 24d posterior (dCMAr) & 28 & $54 \pm 3$ & $55 \pm 2$ & $55 \pm 2$ & $59 \pm 1$ & $62 \pm 2$ & $57 \pm 1$ & $56 \pm 2$ & 7 & 13 & 4 & 2 \\
\hline $23 \mathrm{~d}$ & 33 & $49 \pm 3$ & $49 \pm 3$ & $49 \pm 2$ & $49 \pm 4$ & $53 \pm 3$ & $50 \pm 2$ & $52 \pm 3$ & 0 & 8 & 2 & 6 \\
\hline 23d anterior (CMAd) & 33 & $52 \pm 3$ & $49 \pm 2$ & $51 \pm 2$ & $50 \pm 6$ & $57 \pm 3$ & $52 \pm 3$ & $54 \pm 2$ & 2 & 12 & 2 & 6 \\
\hline SSA dorsal bank & 53 & $46 \pm 3$ & $47 \pm 4$ & $46 \pm 3$ & $55 \pm 4$ & $56 \pm 2$ & $52 \pm 2$ & $54 \pm 4$ & 20 & 22 & 13 & 17 \\
\hline \multicolumn{13}{|l|}{ Cingulate sulcus (ventral bank) } \\
\hline $24 c$ anterior & 33 & $45 \pm 3$ & $46 \pm 5$ & $45 \pm 3$ & $50 \pm 2$ & $50 \pm 3$ & $50 \pm 3$ & $50 \pm 3$ & 11 & 11 & 11 & 11 \\
\hline $24 \mathrm{c}$ middle & 37 & $50 \pm 5$ & $50 \pm 2$ & $49 \pm 3$ & $53 \pm 2$ & $59 \pm 2$ & $54 \pm 3$ & $53 \pm 5$ & 8 & 20 & 10 & 8 \\
\hline 24c posterior (vCMAr) & 28 & $49 \pm 4$ & $51 \pm 5$ & $50 \pm 3$ & $52 \pm 6$ & $54 \pm 6$ & $52 \pm 3$ & $50 \pm 5$ & 4 & 8 & 4 & 0 \\
\hline $23 c(C M A v)$ & 30 & $42 \pm 2$ & $42 \pm 2$ & $42 \pm 2$ & $46 \pm 2$ & $50 \pm 3$ & $45 \pm 1$ & $45 \pm 1$ & 10 & 19 & 7 & 7 \\
\hline SSA ventral bank & 77 & $42 \pm 3$ & $45 \pm 3$ & $44 \pm 4$ & $46 \pm 3$ & $48 \pm 1$ & $46 \pm 1$ & $46 \pm 2$ & 5 & 9 & 5 & 5 \\
\hline \multicolumn{13}{|l|}{ Medial areas (ventral convexity) } \\
\hline 24ab anterior & 51 & $34 \pm 2$ & $34 \pm 2$ & $34 \pm 2$ & $36 \pm 3$ & $38 \pm 2$ & $40 \pm 1$ & $40 \pm 2$ & 6 & 12 & 18 & 18 \\
\hline 24ab posterior & 51 & $39 \pm 4$ & $40 \pm 2$ & $40 \pm 2$ & $40 \pm 2$ & $45 \pm 3$ & $44 \pm 1$ & $43 \pm 2$ & 0 & 13 & 10 & 8 \\
\hline $23 a b$ & 69 & $41 \pm 3$ & $43 \pm 3$ & $42 \pm 3$ & $43 \pm 4$ & $47 \pm 2$ & $43 \pm 3$ & $45 \pm 2$ & 2 & 12 & 2 & 7 \\
\hline 31 & 103 & $42 \pm 2$ & $44 \pm 4$ & $43 \pm 2$ & $42 \pm 2$ & $45 \pm 2$ & $44 \pm 2$ & $46 \pm 2$ & -2 & 5 & 2 & 7 \\
\hline
\end{tabular}

$n$, Number of sets of five adjacent horizontal sections used to obtain the mean LCGU values (in micromoles per $100 \mathrm{~g}$ per min) for each region. (m values represent the average $L C G U$ values from the two hemispheres of the motion-control monkey. El and Er values represent the average LCGU values from the two left and the two right hemispheres of the grasping-execution monkeys, respectively. 01 and 0 r values represent the average $L C G U$ values from the three left and the three right hemispheres of the grasping-observation monkeys, respectively. El/ $\mathrm{Cm}, \mathrm{Er} / \mathrm{Cm}, 0 \mathrm{l} / \mathrm{Cm}$, and $\mathrm{Or} / \mathrm{Cm}$ represent percentage differences between El, Er, $0 \mathrm{l}$, and $0 \mathrm{r}$ and $\mathrm{Cm}$, respectively, calculated as (experimental - control)/ control $\times 100$. Values in bold indicate statistically significant differences by the Student's unpaired $t$ test at the level of $p<0.001$.

addition to the SI-forelimb region and to the distal (but not proximal) forelimb representation in MI/F1 (Fig. 4b, Table 1). Activations were also found in medial cortical areas, such as the F6/ pre-SMA, 9m, 8m, 24, and SSA (Fig. 5b, Table 1). Most of the activations were found to be bilateral (Table 1). The only significant depression that we measured was in the middle portion of $24 \mathrm{~d}$ (dorsal bank of Cgs) in the right hemispheres of both $\mathrm{E}$ and $\mathrm{O}$ cases (Table 1). The absence of increased activity in EMG records of the $\mathrm{O}$ monkeys convinced us that the herein documented cortical effects are not attributable to and do not generate muscular contractions.

When spatially analyzed, the activations induced by observation of grasping movements were found to largely overlap those induced by execution of the same movements within both the lateral (Fig. 4c) and the medial (Fig. 5c) cortical maps of activity. In the latter figures, red, green, and yellow correspond to 
a
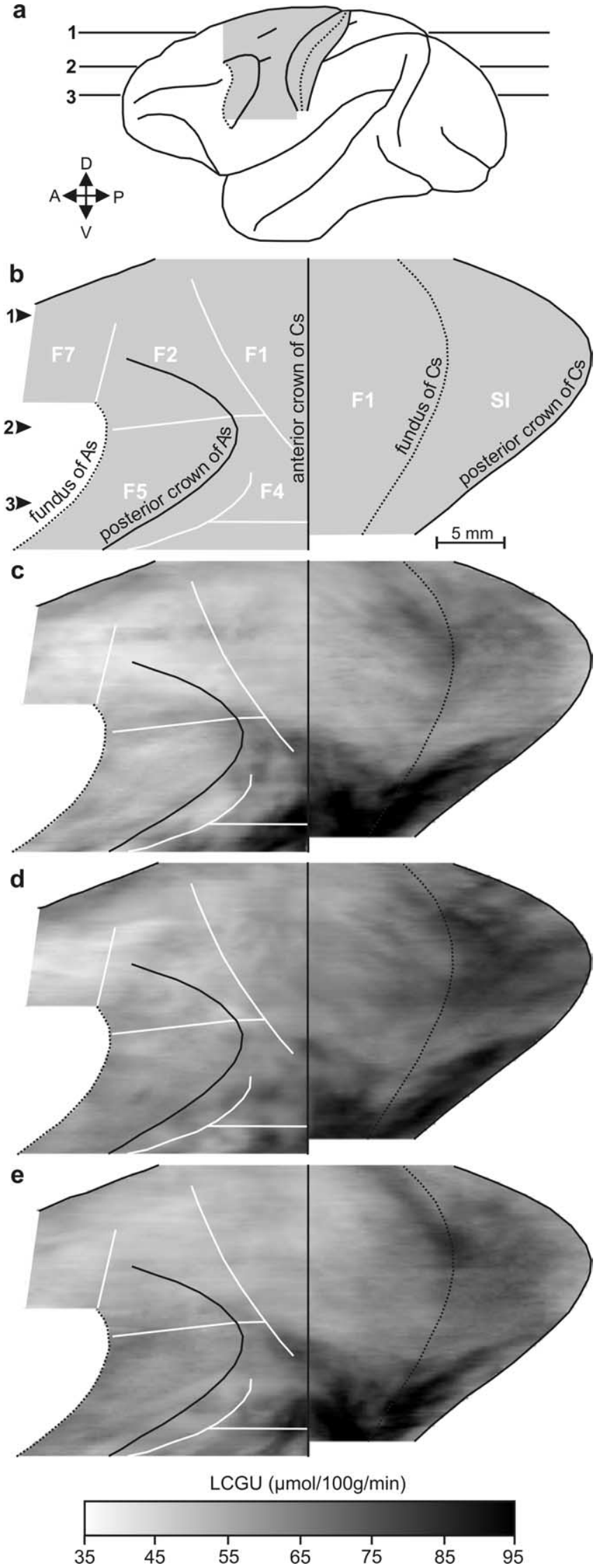

Figure 2. Quantitative 2D maps of metabolic activity in the lateral-frontal cortex. $\boldsymbol{a}$, Lateral view of a monkey brain with the Cs and the posterior bank of the As unfolded. Dotted lines depict the fundus of the As and that of the Cs. Shaded area indicates the reconstructed cortex. Horizontal lines 1-3 correspond to three different dorsoventral levels of brain sectioning. A, Ante- execution-induced, observation-elicited, and common activations, respectively. However, when the intensity of activation was also taken into account, the effects of action execution differed from those of action observation. To graphically illustrate the spatio-intensive distribution of metabolic activity within the affected regions, we plotted the differences between the experimental monkeys and the Cm (as percentage LCGU values and 95\% confidence intervals per $100 \mu \mathrm{m}$ ) across the rostrocaudal extent in the reconstructed maps (Fig. 6). The plots in this figure represent the percentage differences between the $\mathrm{E}$ and the $\mathrm{Cm}$ monkeys (red lines) as well as between the $\mathrm{O}$ and the $\mathrm{Cm}$ monkeys (green lines). Baseline indicates $0 \%$ difference from the $\mathrm{Cm}$. The plots in Figure $6 a$ represent differences in the forelimb representations of the dorsal premotor and the primary sensory-motor cortices along the ribbon highlighted in the brain sketch above the graphs. In the left hemispheres of the two E monkeys ipsilateral to the grasping hand, activity of all cortical areas is similar to that of the corresponding areas in the $\mathrm{Cm}$ (Fig. $6 a$, dotted red line fluctuates around $0 \%)$. In contrast, significantly larger activations were found within the premotor and primary sensorymotor cortices of the hemispheres contralateral to the grasping hand (Fig. $6 a$, solid red line) resulting in a pronounced side-toside difference in the $\mathrm{E}$ monkeys. Interestingly, the corresponding side-to-side difference in the $\mathrm{O}$ monkeys is much smaller (Fig. $6 a$, distance between the solid and the dotted green lines). Consequently, the sensory-motor activations induced by action execution are mostly contralateral to the moving forelimb, in contrast to those elicited by action observation, which are mainly bilateral (see also Table 1). Moreover, in the $\mathrm{O}$ monkeys the primary sensory-motor cortex is less activated, whereas the dorsal premotor is more activated, than the corresponding areas of the affected hemisphere (contralateral to the moving forelimb) of the E monkeys (Fig. $6 a$, Table 1). The plots in Figure $6 b$ represent percentage LCGU differences between the experimental and the Cm monkeys across the rostrocaudal extent of the dorsal bank of the cingulate sulcus. Smaller side-to-side differences and higher anterior (than posterior) activations in the $\mathrm{O}$ relative to the $\mathrm{E}$ monkeys were also found in this case (Fig. 6b) as well as in other medial cortical areas (Table 1).

\section{Discussion}

\section{Effects induced by grasping execution and} grasping observation

Our quantitative high-resolution neuroimaging study combined with cytoarchitectonic identification of cortical areas demonstrates conclusively for the first time the extensive overlap of the action execution and action observation systems within the lateral and medial frontal and cingulate sensory-motor cortical network of primates. Of course, the overlapping activations for action execution and action observation do not necessarily indicate involvement of the same cell populations in the two conditions.

Of the lateral-frontal cortical areas we examined, the distal forelimb representations in MI/F1 and SI of the Cs were activated

\section{$\leftarrow$}

rior; $D$, dorsal; $P$, posterior; $V$, ventral. $\boldsymbol{b}$, Schematic illustration of the geometrically normalized reconstructed cortical field. Black lines correspond to surface landmarks and white lines to cytoarchitectonically identified borders of the labeled cortical areas. Arrows 1-3 indicate the dorsoventral levels of the corresponding lines in $\boldsymbol{a}$. $\boldsymbol{c}$, Averaged map from the two hemispheres of the motion-control monkey. $\boldsymbol{d}$, Averaged map from the right hemispheres (contralateral to the moving forelimb) of the two grasping-execution monkeys. $\boldsymbol{e}$, Averaged map from the right hemispheres of the three grasping-observation monkeys. Grayscale bar indicates LCGU values in micromoles per $100 \mathrm{~g}$ per minute. 
for both execution and observation, in contrast to the proximal forelimb representation in the MI/F1 of the convexity (rostral to the Cs), which was activated only for grasping execution. The F2forelimb representation, the F5-bank, and F5-convexity were involved in both execution and observation of grasping. Our findings confirm previous reports demonstrating that mere observation of goaldirected hand actions modulates activity in the MI/F1 and SI (Hari et al., 1998; Avikainen et al., 2002), the dorsal (Grafton et al., 1996; Decety et al., 1997; Buccino et al., 2001; Cisek and Kalaska, 2004; Filimon et al., 2007), and the ventral premotor (Grafton et al., 1996; Rizzolatti et al., 1996; Decety et al., 1997; Nelissen et al., 2005) cortical areas, which are normally activated by execution of the same hand movements, and facilitates the excitability of the observer's spinal circuitry, which is normally involved in hand movement execution (Fadiga et al., 1995; Maeda et al., 2002; Romani et al., 2005). Our findings also confirm previous studies reporting that the motor circuitry is activated mainly contralaterally to the moving forelimb for action execution (Gregoriou et al., 2005) and bilaterally for action observation (Nishitani and Hari, 2000; Costantini et al., 2005; Filimon et al., 2007). Our finding that F7 is involved in observation, F2 in both observation and execution, and MI/ F1-convexity in execution is compatible with reports that the rostral part of the human premotor cortex is more active during motor imagery and the caudal part during motor execution of hand movements (Gerardin et al., 2000; Lacourse et al., 2005).

Of the medial-frontal and cingulate cortical areas that we examined, F6/preSMA, $8 \mathrm{~m}, 9 \mathrm{~m}$, anterior $24 \mathrm{~d}, 24 \mathrm{c}, 24 \mathrm{ab}$, and SSA were involved in both execution and observation, whereas F3/SMA-proper, CMAs, and area 23 were involved mainly in execution. Our findings confirm previous reports demonstrating that observation and execution of goal-directed hand actions activate in common medialfrontal areas 9 and 6-rostral (Grafton et al., 1996; Decety et al., 1997). Interestingly,

area F6/pre-SMA herein documented to be involved in both action execution and action observation has been associated with effector-independent aspects of motor behavior (Fujii et al., 2002), whereas area F3/SMA-proper herein found to be involved only in action execution is known to project directly to MI/F1 and the spinal cord (He et al., 1995) and to be more closely associated with actual execution (Fujii et al., 2002). Our finding that only the F6/pre-SMA is involved in action observation, whereas both F6/ pre-SMA and F3/SMA-proper are involved in action execution, is consistent with reports of a covert-to-overt rostrocaudal segregaare as in Figure 2.
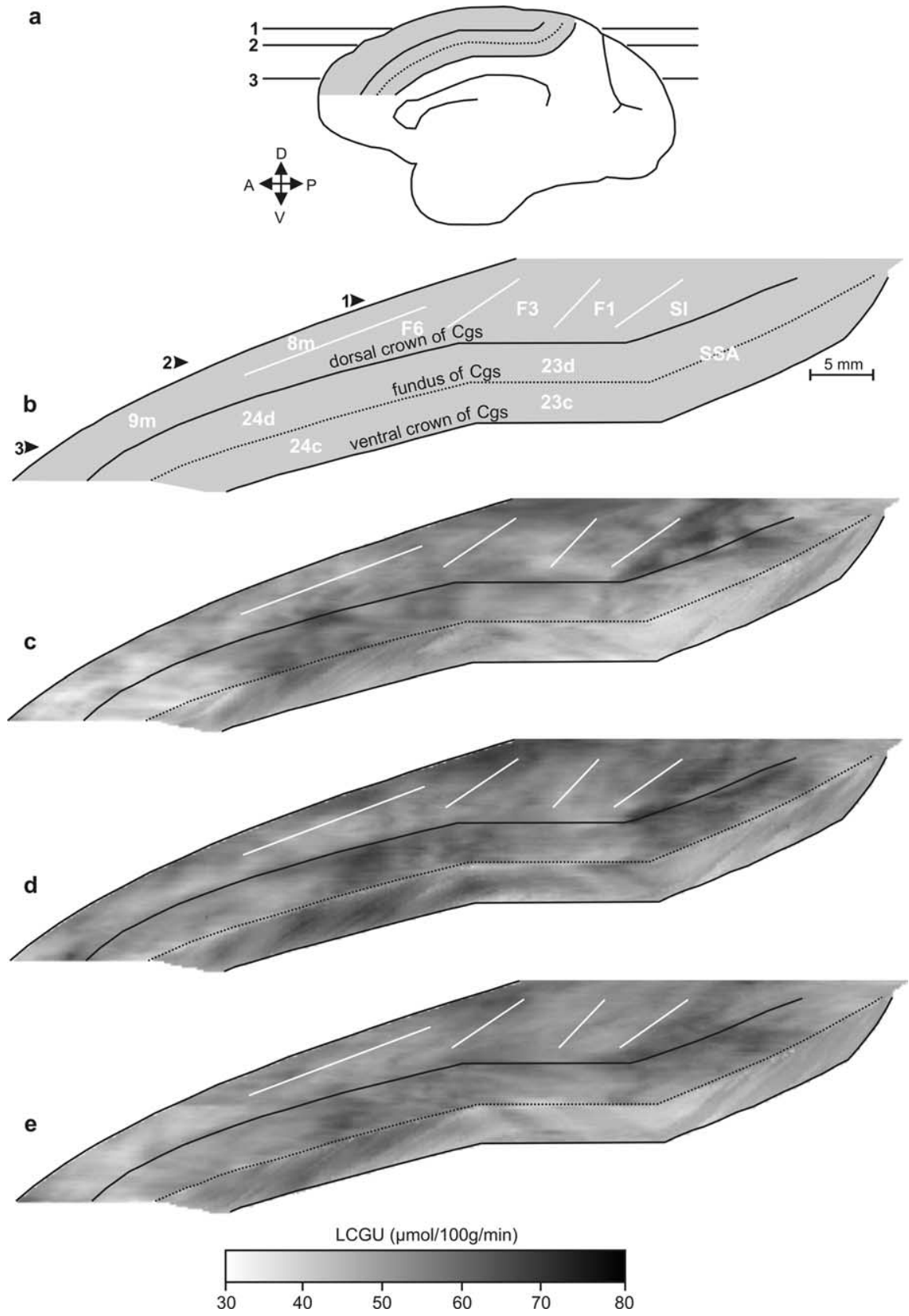

Figure 3. Quantitative 2D maps of metabolic activity in the medial convexity and the Cgs. $\boldsymbol{a}$, Medial view of a monkey brain with the Cgs unfolded. Dotted line depicts the fundus of the sulcus. Shaded area represents the reconstructed cortex. $\boldsymbol{b}$, Schematic illustration of the geometrically normalized reconstructed cortical field. Black lines correspond to surface landmarks and white lines to cytoarchitectonically identified borders of the labeled cortical areas. c, Averaged map from the two hemispheres of the motion-control monkey.d, Averaged map from the right hemispheres (contralateral to the moving forelimb) of the two graspingexecution monkeys. $\boldsymbol{e}$, Averaged map from the right hemispheres of the three grasping-observation monkeys. Other conventions

tion reported in the medial-frontal cortex (Stephan et al., 1995; Decety, 1996; Grafton et al., 1996; Gerardin et al., 2000; Nishitani and Hari, 2000; Costantini et al., 2005; Filimon et al., 2007). Also, our finding that the medial cortical area 9 and the anterior part of area 24 are involved in both execution and observation is compatible with the suggestion that these areas control a goal-based action selection (Matsumoto et al., 2003). Finally, the region in the caudalmost portion of the Cgs, which was activated for both execution and observation of action in our study, corresponds to an area that has been designated as the transitional and supple- 

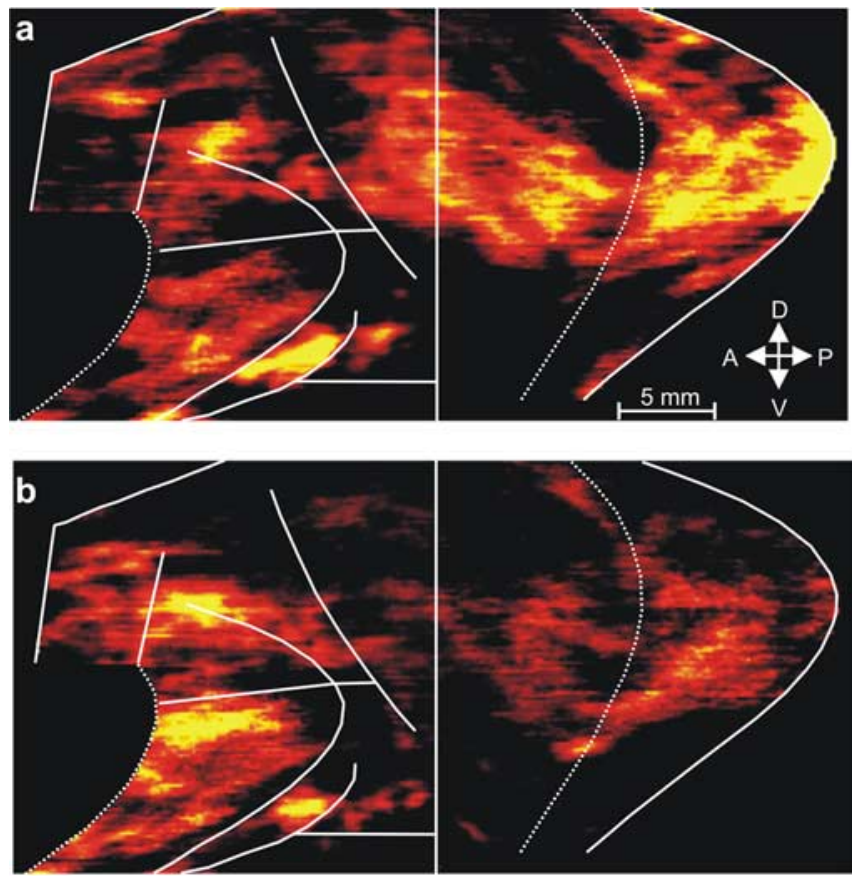

LCGU difference (\%)
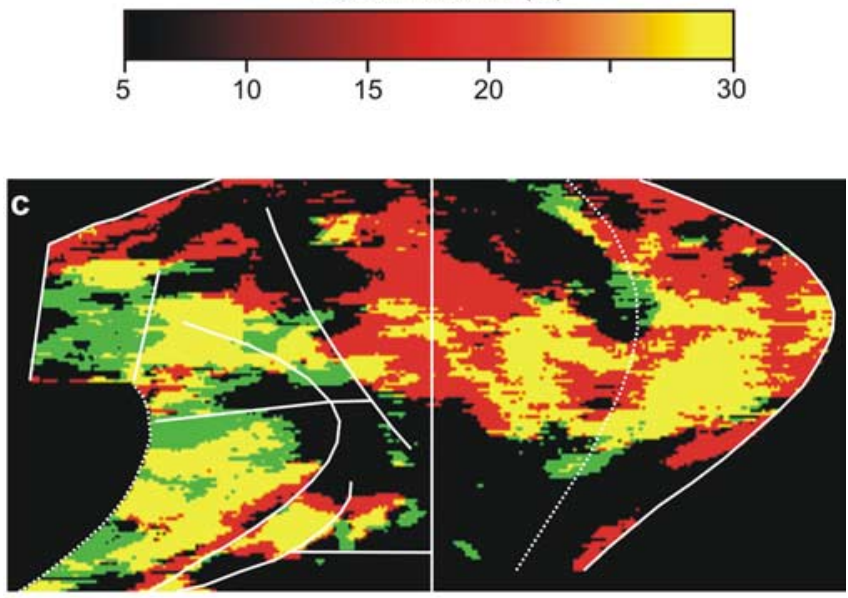

execution

observation

common

Figure 4. Lateral-frontal cortical maps of percentage LCGU differences from the $\mathrm{Cm}$. Percentage differences were calculated using the formula $(\mathrm{E}-\mathrm{Cm}) / \mathrm{Cm} \times 100$ for execution and $(0-\mathrm{Cm}) / \mathrm{Cm} \times 100$ for observation. $\boldsymbol{a}$, Map of net execution-induced activations averaged from the right hemispheres (contralateral to the moving forelimb) of the two graspingexecution monkeys. $\boldsymbol{b}$, Map of net observation-induced activations averaged from the right hemispheres of the three grasping-observation monkeys. Color bar indicates percentage LCGU differences from the $\mathrm{Cm}$. $\boldsymbol{c}$, Superimposition of $\boldsymbol{a}$ and $\boldsymbol{b}$. Red and green represent activations $>10 \%$ induced by grasping execution and grasping observation, respectively. Yellow stands for activations induced by both execution and observation of the same action. White lines correspond to the surface landmarks and the cytoarchitectonic borders illustrated in Figure 2.

mentary somatosensory area (SSA) and that is strongly connected to the sensory-motor specific cortices (Murray and Coulter, 1981; Morecraft et al., 2004).

\section{Mental simulation of actions in the service of action recognition}

The fact that the execution and the observation of the same action rely on a largely common distributed neural system indicates that they are functionally intertwined and substantiates the often-
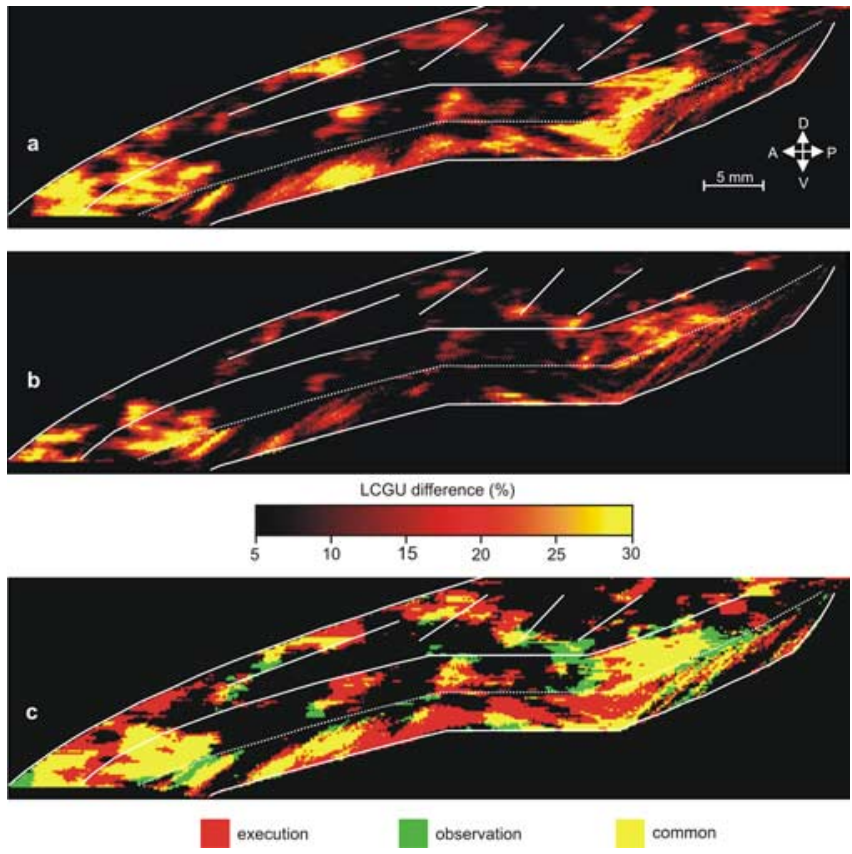

Figure 5. Maps of the medial convexity and the cingulate sulcus: percentage LCGU differences from the Cm. $\boldsymbol{a}$, Map of net execution-induced activations averaged from the right hemispheres (contralateral to the moving forelimb) of the two grasping-execution monkeys. $\boldsymbol{b}$, Map of net observation-induced activations averaged from the right hemispheres of the three grasping-observation monkeys. Color bar indicates percentage LCGU differences from the $\mathrm{Cm}$. c, Superimposition of $\boldsymbol{a}$ and $\boldsymbol{b}$. Red and green represent activations $>10 \%$ induced by grasping execution and grasping observation, respectively. Yellow stands for activations matched for execution and observation. White lines correspond to the surface landmarks and the cytoarchitectonic borders illustrated in Figure 3 .

considered parity between perception and action. Our finding that observing an action excites the motor programs used to execute that same action implies that observation of an action corresponds to simulation of its overt counterpart. Therefore, to recognize the actions of another person and to understand his behavior, the observer may be putting himself in the actor's shoes. This mechanism resembles the internal recital or the mental rehearsal of the observed action. In other words, we could be decoding the actions of others by activating our own action system. We could understand observed actions by executing them "mentally."

It is reasonable to ask why the activation of the motor system during observation of an action does not result in overt movements. As previously proposed, a dual mechanism may operate at the spinal level, involving a subthreshold excitatory corticospinal input (preparation to move) and a parallel inhibitory influence (suppression of overt movement) via the brainstem or the cerebellum (Blakemore et al., 2001; Jeannerod, 2001). The herein documented smaller (by 50\%) activation of the MI/F1-forelimb in the Cs for action observation than for action execution supports the hypothesis that the actions of others are decoded by activating one's own corticospinal system at a subthreshold level. Similarly, our finding that the rostrodorsal and rostroventral lateral premotor cortical activations are higher (by $40 \%$ ) for action observation than for action execution supports the hypothesis that a parallel inhibitory influence may block the overt action. For example, area F7, which is activated for observation but not for execution, may inhibit the $\alpha$-motoneurons via the brainstem (Keizer and Kuypers, 1989). A more detailed picture of the possible underlying inhibitory network(s) will be obtained when the 

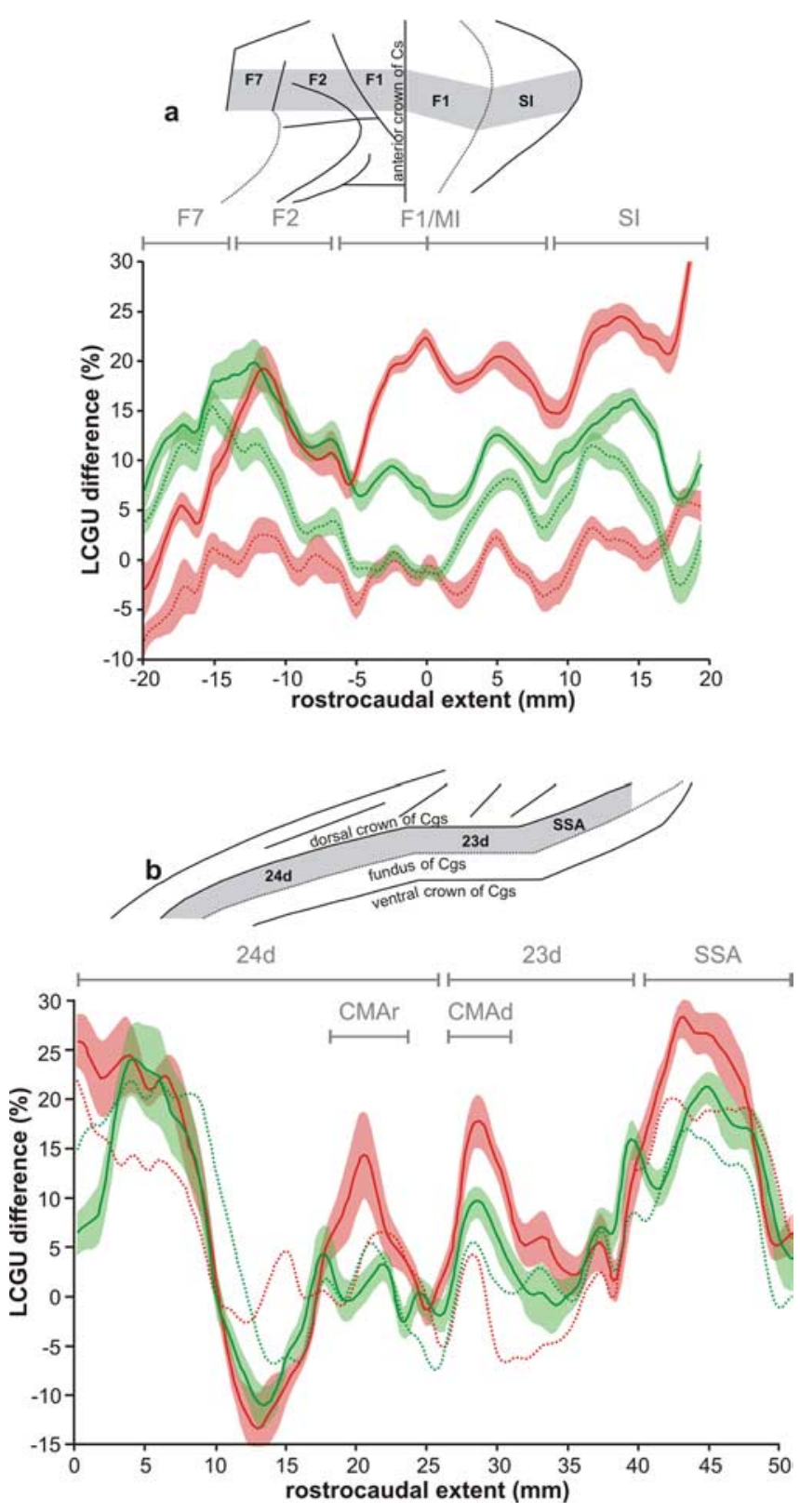

Figure 6. Plots of percentage LCGU differences along the rostrocaudal extent of the reconstructed cortical fields. Red plots illustrate the differences between the two execution monkeys and the $\mathrm{Cm}$. Green plots illustrate the differences between the three observation monkeys and the $\mathrm{Cm}$. Plots with solid and dotted lines correspond to the right and the left hemispheres, respectively. Red and green shaded areas indicate $95 \%$ confidence intervals. Baseline corresponds to $0 \% \mathrm{LCGU}$ difference from the $\mathrm{Cm}$. $\boldsymbol{a}$, Plot along the rostrocaudal extent of the forelimb representations in the dorsal premotor (F7, F2) and the primary sensory-motor (F1/MI, SI) cortices (along the ribbon highlighted in the drawing above the plots). Zero rostrocaudal extent represents the point of alignment of the horizontal brain sections in the lateral-frontal reconstructed maps, i.e., the anterior crown of the Cs. Areas rostral and caudal to the anterior crown of the (s are represented by negative and positive values, respectively. $\boldsymbol{b}$, Plot along the rostrocaudal extent of the dorsal bank of the Cgs, highlighted in the drawing above the plots.

cerebellar and brainstem motor components of our monkeys are analyzed.

It is also reasonable to expect that the simulation of actual movements, which as we suggest underlies action observation, would cause facilitation of their subsequent execution. As a consequence, motor skills could be learned by observation (Mattar and Gribble, 2005). Indeed, the role of observation and imagery (or mental practice) in the teaching of motor skills has already been stated (Hall et al., 1992). Accordingly, we may build our motor repertory by incorporating not only our motor experiences, but also those of other individuals.

All in all, our results undermine the "mirror neuron system" concept. The herein documented fact that the neural correlates of the observation-driven system in the frontal cortex extend well beyond the F5-convexity, where the "mirror neurons" reside (Gallese et al., 1996; Rizzolatti et al., 1996), supports the suggestion that a broader process such as "mental simulation of action" rather than "mirroring" is responsible for action recognition (Goldman and Sebanz, 2005). The concept of mirroring reflects the function of a certain class of cells in premotor area F5 and parietal area PF, which discharge both when a monkey executes an action and when the same monkey observes another subject executing the same action. It thus provides a possible neural substrate for understanding the actions of others. In contrast, the mental simulation theory assigns the role of understanding others' actions to the entire distributed neural network, which is responsible for the execution of actions. In this scenario, action simulation serves the perception of action. Once in place, the neural circuitry that supports action execution can be also used to support the recognition of actions (Goldman and Sebanz, 2005). Neural networks that originally evolved to generate actions ran off-line (decoupled from actual movement) to help understand the actions of others. Accordingly, motor cognition is embodied in action, a notion that supports perceptual (rather than amodal) theories of knowledge claiming that sensory-motor simulators implement fully functional conceptual systems (Barsalou, 1999).

\section{Attribution of action to the correct agent}

The activations induced by observation of grasping movements in anterior premotor areas of the lateral-frontal cortices were stronger than those induced by execution of grasping. In contrast, the activation induced by observation of grasping in the MI/SI-forelimb area was weaker than that induced by execution of grasping. Also, the effects induced by action observation were mainly bilateral, whereas those induced by action execution were mostly contralateral to the moving forelimb. These differential activations of premotor and primary sensory-motor cortices could play a role in attributing the action to the correct agent, i.e., to the other agent during action observation and to the self during action execution. For example, the higher level of MI/FIforelimb cortical activity for action execution may reflect the intended movement (input from the activated premotor cortex) and the actual motor command (MI/F1 cellular activity), whereas the $50 \%$ lower activity for action observation may reflect the intended movement only. Also, the higher level of SI-forelimb cortical activity for action execution may reflect the anticipated sensory consequence of the movement (based on efference copy from MI/F1) and the actual afferent feedback (signal from the muscles), whereas the $50 \%$ lower activity for action observation may reflect the anticipated consequence of the movement only. Furthermore, although there is no direct evidence from our data, we suggest that the bilateral and more intense (dorsal and ventral) lateral premotor cortical activations for action observation could reflect the discrepancy between the programmed (represented) movement and the lack of the corresponding sensory feedback. In other words, they could reflect the incongruence between the predicted consequence of the triggered movement representation and the lack of its actual afferent feedback during mere observation. Interestingly, the experience of ourselves or others as the cause of an action may derive from the ability to compare motor commands with reafference from the body 
movements and external events caused by the commands (Johnson and Haggard, 2005). There are several reports assigning to the anterior section of the frontal cortex a role in sense of agency (feeling in control over a sensory event linked to one's own action), action attribution (deciding which action belongs to which agent), and mental-state ascription [for review, see Decety and Sommerville (2003) and Gallagher and Frith (2003)]. However, our findings suggest that the anterior frontal cortical areas associated with attribution of action in the abovementioned studies may constitute central components of the execution/perception distributed sensory-motor circuit rather than extra machinery functioning on a side path of this circuitry.

\section{References}

Avikainen S, Forss N, Hari R (2002) Modulated activation of the human SI and SII cortices during observation of hand actions. NeuroImage 15:640-646.

Barsalou LW (1999) Perceptual symbol systems. Behav Brain Sci 22:577-609.

Blakemore SJ, Frith CD, Wolpert DW (2001) The cerebellum is involved in predicting the sensory consequences of action. NeuroReport 12:1879-1885.

Buccino G, Binkofski F, Fink GR, Fadiga L, Fogassi L, Gallese V, Seitz RJ, Zilles K, Rizzolatti G, Freund HJ (2001) Action observation activates premotor and parietal areas in a somatotopic manner: an fMRI study. Eur J Neurosci 13:400-404.

Cisek P, Kalaska JF (2004) Neural correlates of mental rehearsal in dorsal premotor cortex. Nature 431:993-996.

Costantini M, Galati G, Ferretti A, Caulo M, Tartaro A, Romani GL, Aglioti SM (2005) Neural systems underlying observation of humanly impossible movements: an FMRI study. Cereb Cortex 15:1761-1767.

Decety J (1996) Do imagined and executed actions share the same neural substrate? Cogn Brain Res 3:87-93.

Decety J, Sommerville JA (2003) Shared representations between self and other: a social cognitive neuroscience view. Trends Cogn Sci 7:527-533.

Decety J, Grezes J, Costes N, Perani D, Jeannerod M, Procyk E, Grassi F, Fazio F (1997) Brain activity during observation of actions. Influence of action content and subject's strategy. Brain 120:1763-1777.

Fadiga L, Fogassi L, Pavesi G, Rizzolatti G (1995) Motor facilitation during action observation: a magnetic stimulation study. J Neurophysiol 73:2608-2611.

Filimon F, Nelson JD, Hagler DJ, Sereno MI (2007) Human cortical representations for reaching: mirror neurons for execution, observation, and imagery. NeuroImage 37:1315-1328.

Fujii N, Mushiake H, Tanji J (2002) Distribution of eye- and armmovement-related neuronal activity in the SEF and in the SMA and preSMA of monkeys. J Neurophysiol 87:2158-2166.

Gallagher HL, Frith CD (2003) Functional imaging of "theory mind." Trends Cogn Sci 7:77-83.

Gallese V, Fadiga L, Fogassi L, Rizzolatti G (1996) Action recognition in the premotor cortex. Brain 119:593-609.

Gerardin E, Sirigu A, Lehericy S, Poline JB, Gaymard B, Marsault C, Agid Y, Bihan D (2000) Partially overlapping neural networks for real and imagined hand movements. Cereb Cortex 10:1093-1104.

Geyer S, Matelli M, Luppino G, Zilles K (2000) Functional neuroanatomy of the primate isocortical motor system. Anat Embryol 202:443-474.

Goldman AI, Sebanz N (2005) Simulation, mirroring, and a different argument from error. Trends Cogn Sci 9:320.

Grafton ST, Arbib MA, Fadiga L, Rizzolatti G (1996) Localization of grasp representations in humans by positron emission tomography. 2. Observation compared with imagination. Exp Brain Res 112:103-111.

Gregoriou GG, Luppino G, Matelli M, Savaki HE (2005) Frontal cortical areas of the monkey brain engaged in reaching behavior: a (14)Cdeoxyglucose imaging study. NeuroImage 27:442-464.

Hall C, Buckolz E, Fishburne GJ (1992) Imagery and the acquisition of motor skills. J Can Sport Sci 17:19-27.

Hari R, Forss N, Avikainen S, Kirveskari E, Salenius S, Rizzolatti G (1998) Activation of human primary motor cortex during action observation: a neuromagnetic study. Proc Natl Acad Sci USA 95:15061-15065.

He S-Q, Dum RP, Strick PL (1995) Topographic organization of corticospinal projections from the frontal lobe: motor areas on the medial surface of the hemisphere. J Neurosci 15:3284-3306.

Jeannerod M (2001) Neural simulation of action: a unifying mechanism for motor cognition. NeuroImage 14:S103-S109.

Johnson H, Haggard P (2005) Motor awareness without perceptual awareness. Neuropsychologia 43:227-237.

Keizer K, Kuypers HGJM (1989) Distribution of corticospinal neurons with collaterals to the lower brain stem reticular formation in monkey. Exp Brain Res 74:311-318.

Kennedy C, Sakurada O, Shinohara M, Jehle J, Sokoloff L (1978) Local cerebral glucose utilization in the normal conscious macaque monkey. Ann Neurol 4:293-301.

Lacourse MG, Orr EL, Cramer SC, Cohen MJ (2005) Brain activation during execution and motor imagery of novel and skilled sequential hand movements. NeuroImage 27:505-519.

Maeda F, Kleiner-Fisman G, Pascual-Leone A (2002) Motor facilitation while observing hand actions: specificity of the effect and role of observer's orientation. J Neurophysiol 87:1329-1335.

Matelli M, Luppino G, Rizzolatti G (1991) Architecture of superior and mesial area 6 and the adjacent cingulate cortex in the macaque monkey. J Comp Neurol 311:445-462.

Matsumoto K, Suzuki W, Tanaka K (2003) Neuronal correlates of goalbased motor selection in the prefrontal cortex. Science 301:229-232.

Mattar AA, Gribble PL (2005) Motor learning by observing. Neuron 46:153-160.

Morecraft RJ, Cipolloni PB, Stilwell-Morecraft KS, Gedney MT, Pandya DN (2004) Cytoarchitecture and cortical connections of the posterior cingulate and adjacent somatosensory fields in the rhesus monkey. J Comp Neurol 469:37-69.

Murray EA, Coulter JD (1981) Supplementary sensory area. The medial parietal cortex in monkey. Clifton, NJ: Humana.

Nelissen K, Luppino G, Vanduffel W, Rizzolatti G, Orban GA (2005) Observing others: multiple action representation in the frontal lobe. Science 310:332-336.

Nishitani N, Hari R (2000) Temporal dynamics of cortical representation for action. Proc Natl Acad Sci USA 97:913-918.

Raos V, Evangeliou MN, Savaki HE (2004) Observation of action: grasping with the mind's hand. NeuroImage 23:193-201.

Rizzolatti G, Fadiga L, Gallese V, Fogassi L (1996) Premotor cortex and the recognition of motor actions. Cogn Brain Res 3:131-141.

Romani M, Cesari P, Urgesi C, Facchini S, Aglioti SM (2005) Motor facilitation of the human cortico-spinal system during observation of biomechanically impossible movements. NeuroImage 26:755-763.

Savaki HE, Kennedy C, Sokoloff L, Mishkin M (1993) Visually guided reaching with the forelimb contralateral to a "blind" hemisphere: a metabolic mapping study in monkeys. J Neurosci 13:2772-2789.

Sokoloff L, Reivich M, Kennedy C, Des Rosiers MH, Patlak CS, Pettigrew KS, Sakurada O, Shinohara M (1977) The $\left[{ }^{14} \mathrm{C}\right]$-deoxyglucose method for the measurement of local cerebral glucose utilization: theory, procedure, and normal values in the conscious and anesthetized albino rat. J Neurochem 28:879-916.

Stephan KM, Fink GR, Passingham RE, Silbersweig D, Ceballos-Baumann AO, Frith CD, Frackowiak RS (1995) Functional anatomy of the mental representation of upper extremity movements in healthy subjects. J Neurophysiol 73:373-386. 